\title{
White matter volume changes in people who develop psychosis
}

Mark Walterfang, Philip K. McGuire, Alison R. Yung, Lisa J. Phillips, Dennis Velakoulis, Stephen J. Wood, John Suckling, Edward T. Bullmore, Warrick Brewer, Bridget Soulsby, Patricia Desmond, Patrick D. McGorry and Christos Pantelis

\section{Background}

Grey matter changes have been described in individuals who are pre- and peri-psychotic, but it is unclear if these changes are accompanied by changes in white matter structures.

\section{Aims}

To determine whether changes in white matter occur prior to and with the transition to psychosis in individuals who are pre-psychotic who had previously demonstrated grey matter reductions in frontotemporal regions.

\section{Method}

We used magnetic resonance imaging (MRI) to examine regional white matter volume in 75 people with prodromal symptoms. A subset of the original group $(n=21)$ were rescanned at 12-18 months to determine white matter volume changes. Participants were retrospectively categorised according to whether they had or had not developed psychosis at follow-up.

\section{Results}

Comparison of the baseline MRI data from these two subgroups revealed that individuals who later developed psychosis had larger volumes of white matter in the frontal lobe, particularly in the left hemisphere. Longitudinal comparison of data in individuals who developed psychosis revealed a reduction in white matter volume in the region of the left fronto-occipital fasciculus. Participants who had not developed psychosis showed no reductions in white matter volume but increases in a region subjacent to the right inferior parietal lobule.

\section{Discussion}

The reduction in volume of white matter near the left frontooccipital fasciculus may reflect a change in this tract in association with the onset of frank psychosis.

\section{Declaration of interest} None.
There is a consensus that there are radiologically detectable volumetric abnormalities in schizophrenia and bipolar disorder, ${ }^{1}$ and that these are apparent at the time of the onset of frank psychosis. Whether these changes progress over time has been more a contentious issue ${ }^{2}$ but recent longitudinal magnetic resonance imaging (MRI) studies suggest that there are progressive reductions in global and regional grey matter volume. ${ }^{3-7}$ Furthermore, there is some evidence that progressive changes may occur before the onset of psychosis. We previously found that in people at ultrahigh risk of developing psychosis who subsequently developed psychosis, there was a longitudinal reduction in grey matter volume in the inferior frontal, medial temporal and cingulate cortices between baseline and follow-up scans. ${ }^{8}$ These changes were not evident in individuals who were ultra-high risk who had not developed psychosis at follow-up; similar changes in frontal zones have been reported by other groups and may be predictive of transition to psychosis. ${ }^{9,10}$

Schizophrenia has been postulated to involve disconnectivity on theoretical grounds, ${ }^{11,12}$ and this is consistent with data from functional imaging studies focusing on abnormal correlations between activity in prefrontal and temporal cortices. ${ }^{13}$ Whether there are anatomical correlates of frontotemporal disconnectivity remains in question, although focal reductions in prefrontal ${ }^{14-20}$ and temporal ${ }^{21,22}$ white matter regions have been reported. The development of diffusion tensor imaging provides a potentially powerful means of examining anatomical connectivity between different regions of association cortex,${ }^{23,24}$ although to date its application in schizophrenia has produced inconsistent findings. Nevertheless, diffusion tensor imaging studies in schizophrenia have suggested reduced integrity in the uncinate, arcuate, fronto-occipital and superior longitudinal fasciculi, ${ }^{24}$ the major association tracts between higher cortical regions, suggesting alterations to the integrity of these tracts in schizophrenia.
The aim of the present study was to use MRI to assess whether there were white matter abnormalities in people at ultra-high risk of psychosis and whether these were associated with the subsequent development of psychosis. Individuals at ultra-high risk were scanned and then followed clinically for 12 months, during which period a proportion developed psychosis. These individuals were scanned again at the time, whereas those who did not become psychotic were scanned again only at the end of the follow-up period. We tested the hypothesis that those people who developed psychosis would have less white matter in areas carrying fibres between the frontal and temporal lobes, particularly in the left hemisphere, compared with those who did not. A further prediction was that the transition to psychosis would be associated with a further longitudinal reduction in volume in these regions of white matter.

\section{Method}

\section{Participants}

Cross-sectional (baseline) comparison

Seventy-five individuals identified as being at ultra-high risk of developing psychosis were recruited from clients of the Personal Assessment and Crisis Evaluation (PACE) clinic in Melbourne, Australia, which manages young people at risk of developing a psychotic illness. ${ }^{25}$ These represent the subset of individuals scanned with the same dual echo sequence, providing simultaneously acquired $\mathrm{T}_{2}$ and proton density MRI scans.

Selection criteria for the ultra-high risk group have been described elsewhere ${ }^{26}$ and are briefly summarised in the online Table DS1. This sample constituted the entire clinical population of the PACE clinic who consented to be scanned and who met our inclusion criteria, including adequate clinical follow-up in order 
to confirm diagnostic outcome. All participants were aged between 14 and 30 years and had never experienced an episode of frank psychosis. Individuals were excluded if they had a history of head injury, seizures, cerebrovascular disease, other neurological disease, impaired thyroid function or steroid misuse. All participants were assessed with the Brief Psychiatric Rating Scale (BPRS), Scale for the Assessment of Negative Symptoms (SANS) and National Adult Reading Test (NART) at intake (Table DS2).

Participant and instrument details have been provided previously. ${ }^{8}$ Briefly, 23 people $(31 \%)$ developed psychosis over the follow-up interval, 18 within 12 months and a further 5 within 24 months (psychosis group). The remaining 52 (non-psychosis group) did not develop psychosis over the follow-up period, which was at least 12 months ( $89 \%$ reassessed between 12 and 18 months; maximum follow-up 44 months). The groups did not differ in terms of gender, baseline BPRS, SANS or NART score, duration of symptoms or total corrected brain volume. However, the psychosis group was significantly younger $(t(73)=-2.7, P=0.009)$.

In the 23 patients who developed psychosis, the type of psychotic disorder was assessed using the Structured Clinical Interview for DSM-IV (SCID). The diagnostic breakdown in these patients was: schizophrenia $(n=8)$, schizoaffective disorder $(n=2)$, brief psychotic episode $(n=1)$, psychosis not otherwise specified $(n=1)$, bipolar disorder with psychotic features $(n=6)$ and major depression with mood incongruent psychotic disorder $(n=4)$; 1 patient had recently developed a psychosis with affective features (SCID diagnosis unavailable). Twenty-eight individuals (54\%) who did not develop psychosis had no psychiatric diagnosis at follow-up. Of those who met criteria for a psychiatric disorder, 11 had a mood disorder (5 with major depressive disorder; 6 with dysthymia); 8 had an anxiety disorder (4 social phobia; 2 general anxiety disorder; 2 panic disorder); and 3 had obsessive-compulsive disorder.

\section{Longitudinal study}

Twenty-one of the original 75 participants were rescanned using the same MRI protocol as at baseline and represented all those in whom follow-up scanning was possible. These individuals were representative of the larger pool of participants scanned at baseline on all demographic and clinical variables (age, height, IQ, duration of symptoms, BPRS and SANS ratings) apart from gender (more females than in the larger group; $\chi^{2}=6.25, P=0.012$ ), and did not differ for whole brain volume. Ten of the 21 participants developed psychosis (psychosis group) during the follow-up period and 11 did not (non-psychosis group) (Table DS2). It was intended that all people who entered the psychosis group would be scanned as soon as possible after the onset of their psychosis, whereas those who remained in the non-psychosis group would be scanned after the 12-month follow-up period had elapsed. However, in practice it was not always possible to contact these patients at the desired time, resulting in a large range for the inter-scan interval. Most participants in both groups did not receive antipsychotic medication before psychosis onset (psychosis group) or by time of second scan (non-psychosis group), except for three individuals in the non-psychosis group and two psychosis group, who received 1-2 mg risperidone for part of this period. Following the onset of psychosis, all patients but one received atypical antipsychotic medication (nine patients were treated with 1-3 mg risperidone; one patient received trifluoperazine; one risperidone-treated patient was non-adherent).

The diagnostic breakdown (as defined using the SCID) of patients in the psychosis subgroup who were scanned at followup was; schizophrenia $(n=5)$, schizoaffective disorder $(n=1)$, brief psychotic episode $(n=1)$, psychosis not otherwise specified $(n=1)$, manic episode with psychotic features $(n=1)$ and major depression with mood incongruent psychotic disorder $(n=1)$. Of the 11 patients in the non-psychosis group who were rescanned at follow-up, 6 had no psychiatric diagnosis, and the remaining 5 patients were diagnosed with major depressive disorder, dysthymia, generalised anxiety disorder, eating disorder and obsessive-compulsive disorder respectively.

\section{Structural MRI}

\section{Image acquisition}

High-resolution $3 \mathrm{~mm}$ thick, contiguous, interleaved twodimensional dual echo fast spin echo images were acquired parallel to the anterior commissure-posterior commissure line, covering the entire brain. All participants were scanned on a GE Signa 1.5 T scanner at a single site. Proton density-weighted and $\mathrm{T}_{2}$-weighted images were acquired almost simultaneously (echo time $(\mathrm{TE})_{1}=20 \mathrm{~ms}, \mathrm{TE}_{2}=85 \mathrm{~ms}$, repetition time $(\mathrm{TR})=4000 \mathrm{~ms}, 8-$ echo train length). The matrix size and field of view (FOV) were set at $256 \times 192$, and $22 \mathrm{~cm}$ respectively, and each voxel was represented with a 16-bit integer value.

\section{Image processing and analysis}

The methods used for segmentation and registration of each image data-set have been described in detail elsewhere ${ }^{27,28}$ but were briefly as follows. Voxels representing extracerebral tissue were automatically identified and set to zero using a linear scale space set of features obtained from derivatives of the Gaussian kernel. Manual editing of the segmented images was necessary only to remove brain stem from the cerebral hemispheres and diencephalon. The probability of each intracerebral voxel belonging to each of four possible tissue classes (grey matter, white matter, cerebrospinal fluid, or dura/vasculature) was then estimated by a modified fuzzy clustering algorithm. ${ }^{28}$ This algorithm was applied via a 'sliding window' to the images so that classification was adaptive to local variation in tissue contrast due to radio frequency or static field inhomogeneity, which can particularly be a problem in the cerebellar region. ${ }^{28}$ Based on previous findings, we assumed that the resulting probabilities of tissue class membership could be equated with the proportional volumes of each tissue class in the often heterogeneous volume of tissue represented by each voxel. ${ }^{28}$ So, for example, if the probability of grey matter class membership was 0.8 for a given voxel, then it was assumed that $80 \%$ of the tissue represented by that voxel was grey matter. Voxel probabilities of tissue class membership were summed across all voxels and multiplied by the spatial dimensions of each voxel to estimate the total volume of grey matter, white matter and cerebrospinal fluid for each participant.

To allow estimation of between-group structural differences and within-group changes over time at each intracerebral voxel, the proton density-weighted images from each fast spin echo data-set were first co-registered with a template image in the standard space of Talairach and Tournoux by an affine transformation, implemented using the Fletcher-Davidson-Powell algorithm. The template image was constructed by registering each of the images acquired from a subset of the comparison participants in this study in standard space by an affine transformation, and then averaging these images. The affine transformation matrix that mapped each person's proton densityweighted image onto this template image was then applied identically to each of that individual's four tissue class probability maps to register them in standard space at the same voxel size as the original acquisition.

Between-group differences in white matter volume were estimated by fitting an analysis of covariance (ANCOVA) model 
at each intracerebral voxel in standard space that included age at scan (to two decimal places) and global white matter volume as covariates. Within-group differences in white matter were assessed using repeated-measures ANCOVA, also covarying for global white matter volume and for time between scans (also to two decimal places). In both cases, we modelled the effects of the covariates as simple linear effects on the grounds of parsimony. The null hypothesis was tested by permutation at cluster level, as described in detail elsewhere. ${ }^{27}$ In brief, a map of the standardised ANCOVA model coefficient of interest $\beta$ at each voxel was thresholded such that if $\beta>2$ (probability $(\beta)<0.05$, approximately) the voxel value was set to $\beta-2$, otherwise the voxel value was set to zero. This procedure generates a set of suprathreshold voxel clusters in three dimensions, each of which can be described in terms of its mass or the sum of suprathreshold voxel statistics it comprises. The mass of each cluster was tested against a null distribution ascertained by repeatedly randomly permuting the relevant factor in the ANCOVA model; refitting the model and estimating standardised coefficients at each voxel; and thresholding the permuted coefficient maps to generate a set of three-dimensional (3-D) suprathreshold voxel clusters under the null hypothesis. For the first, cross-sectional analysis, the permuted factor coded those patients who would later develop psychosis $v$. those who would not; for the second, longitudinal analysis, the permuted factor coded the data acquired at baseline $v$. the data acquired at follow-up, in the subgroup of patients who developed psychosis over the course of the study.

The rationale for this non-parametric mode of inference is that test statistics for image analysis which incorporate spatial information, such as 3-D cluster mass, are generally more powerful than other possible test statistics, such as $\beta$, which are informed only by data at a single voxel. Statistical inference at the level of voxel clusters has the additional advantage that the number of clusters to be tested (in the order of 100) is considerably less than the number of voxels (in the order of 10000 ) thereby mitigating considerably the severity of the multiple comparisons problem. Yet theoretical approximations to the null distribution of spatial statistics estimated in imaging data may be over-conservative or intractable, ${ }^{27}$ motivating the use of non-parametric or data resampling-based methods to ascertain the null distribution of the cluster statistic by repeated random permutations of the observed data. ${ }^{29,30}$ Here, we have consistently used probability thresholds for cluster-wise testing such that the expected number of false-positive tests for each map is less than one; thus, if the number of clusters to be tested $V=100$, we have applied a cluster-wise probability threshold $P<0.01$ so that the expected number of false-positive tests $P V<1$. Significant foci were anatomically localised using the standard atlas of Talairach and Tournoux, except for foci close to the cerebellum. In the case of the latter foci, we employed the atlas of Schmahmann et $a l^{31}$ as the Talairach atlas lacks anatomical detail in the cerebellar region.

\section{Results}

\section{Cross-sectional analysis}

At baseline, there were significant volumetric differences between participants according to their clinical outcome at follow-up (cluster $P<0.01$ ). Relative to individuals who did not later develop psychosis, the subgroup that subsequently developed psychosis had a larger volume of white matter in an area subjacent to the left premotor cortex, close to the superior fronto-occipital fasciculus, with a trend for a greater volume in a homologous region of white matter in the right hemisphere (cluster $P<0.05$ ) (see online Fig. DS1; Table 1). They also showed a greater volume in a region of white matter adjacent to the left frontal operculum and close to the superior longitudinal fasciculus, and a trend for a greater volume in an area near the right parietal operculum. There were no areas of significantly smaller volume in the subgroup that later developed psychosis, although there was a trend in the right posterior cerebellar hemisphere and in the occipital white matter, near the left optic radiation and the left inferior occipito-temporal fasciculus.

\begin{tabular}{|c|c|c|c|c|c|}
\hline \multirow[b]{2}{*}{ Area } & \multirow[b]{2}{*}{ Left/right } & \multicolumn{3}{|c|}{ Talairach coordinates } & \multirow[b]{2}{*}{ Size, voxels } \\
\hline & & $x$ & $y$ & $z$ & \\
\hline \multicolumn{6}{|l|}{ Reductions } \\
\hline Posterior lobe of cerebellum & $\mathrm{R}$ & 20 & -66 & -28 & 102 \\
\hline Optic radiation & $L$ & -16 & -74 & 10 & 105 \\
\hline \multicolumn{6}{|l|}{ Increases } \\
\hline Lateral to superior longitudinal fasciculus & $\mathrm{R}$ & 43 & -21 & 23 & 66 \\
\hline Superior longitudinal fasciculus & $\mathrm{L}$ & -29 & 3 & 27 & 107 \\
\hline Superior fronto-occipital fasciculus & $\mathrm{L}$ & -27 & -16 & 31 & 175 \\
\hline Superior fronto-occipital fasciculus & $\mathrm{R}$ & 30 & -13 & 31 & 95 \\
\hline
\end{tabular}

Table 2 Talairach coordinates and regions for the significant changes in the patient groups over the follow-up interval

\begin{tabular}{|c|c|c|c|c|c|}
\hline \multirow[b]{2}{*}{ Area } & \multirow[b]{2}{*}{ Left/right } & \multicolumn{3}{|c|}{ Talairach coordinates } & \multirow[b]{2}{*}{ Size, voxels } \\
\hline & & $x$ & $y$ & $z$ & \\
\hline \multicolumn{6}{|l|}{ Psychosis group } \\
\hline \multicolumn{6}{|l|}{ Reductions } \\
\hline Optic radiation & $\mathrm{R}$ & 5 & -67 & 11.5 & 114 \\
\hline Fronto-occipital fasciculus & $\mathrm{L}$ & -23 & -37 & 18 & 78 \\
\hline Inferior part of cerebellum & $\mathrm{L}$ & -21 & -69 & -36 & 102 \\
\hline Inferior part of cerebellum & $\mathrm{R}$ & 8 & -42 & -40 & 153 \\
\hline \multicolumn{6}{|l|}{$\begin{array}{l}\text { Non-psychosis group } \\
\text { Increases }\end{array}$} \\
\hline Cerebellum & $\mathrm{L}$ & -22 & -63 & -42 & 72 \\
\hline Superior longitudinal fasciculus & $\mathrm{R}$ & 30 & -39 & 25 & 146 \\
\hline
\end{tabular}




\section{Longitudinal analysis}

\section{Changes in those who developed psychosis}

Compared to when they had prodromal symptoms, the subgroup of participants that developed psychosis showed a significant reduction $(P<0.01)$ in the white matter deep in the left parietal lobe, near the left fronto-occipital fasciculus (Fig. DS2; Table 2). In addition, there was another region of volume reduction in the occipital lobe, subjacent to the left calcarine cortex. Conversely, after the onset of psychosis, there was an increase in the volume of white matter in the posterior part of the cerebellum bilaterally.

\section{Changes in those who did not develop psychosis}

There were no regional reductions in white matter volume in the participants that did not progress to psychosis. However, there was an increase in white matter volume in the posterior part of the left cerebellum and in an area subjacent to the right inferior parietal lobule (Fig. DS3; Table 2).

\section{Discussion}

The present study used MRI to examine regional white matter volume in a group of individuals at high risk for psychosis because they had an 'at-risk mental state'. We found that within this group, participants who subsequently went on to develop psychosis differed from those who did not, despite these subgroups being clinically indistinguishable at the time of the baseline scans. Furthermore, within-person comparisons of baseline and follow-up scans revealed that there were also longitudinal changes in regional white matter volume, particularly in the subgroup that developed psychosis.

\section{Cross-sectional changes}

A previous analysis of the grey matter from the same participants as in the present study indicated that the psychosis subgroup had smaller grey matter volumes in the inferior frontal, cingulate and medial temporal cortices at baseline. ${ }^{8}$ On the basis of these data, and the literature on abnormal fronto-temporal connectivity in schizophrenia, our first hypothesis was that there would be a reduction in the volume of white matter in regions carrying fibres between the frontal and temporal lobes. Although we found that the subgroup that later developed psychosis differed from those that did not with regard to the volume of white matter in frontal lobe regions bilaterally, the white matter volume was larger rather than smaller. It is difficult to precisely localise these differences, but they were evident close to the superior longitudinal and the fronto-occipital fasciculi, tracts which carry connections between prefrontal and the temporal, parietal and occipital cortices. ${ }^{32-35}$ However, the long axis of some of the statistical clusters was medio-lateral, following that of the white matter subjacent to the frontal cortex. Thus, these differences may have been in fibres connected to the local frontal grey matter, which could have contributed to other tracts as well those connecting the anterior and posterior parts of the hemisphere. Diffusion tensor imaging can provide more detailed information about the tracts within white matter, and the application of tractography to the frontal regions identified in the present study might clarify which particular pathways showed the volumetric differences.

\section{Relationship between grey and white matter changes}

In our earlier study of grey matter volume in the same participants, we found that the subgroup destined to develop psychosis had less grey matter in the inferior frontal and cingulate cortices. ${ }^{8}$ The differences in inferior frontal grey matter volume were primarily in the right hemisphere and at a lower axial level than those in the frontal white matter. Moreover, the differences in frontal white matter were bilateral. The grey matter differences in the cingulate cortex were bilateral and mainly in the posterior part of the gyrus at a similar axial level to the frontal white matter differences. The differences in frontal white matter volume may thus have involved tracts connecting frontal and cingulate cortices, areas that are strongly interconnected, particularly within the same hemisphere. ${ }^{36,37}$ The present study revealed larger white matter volumes in the subgroup who later developed psychosis, whereas our previous study found that the same individuals had relatively smaller frontal and cingulate grey matter volumes than those who did not develop psychosis. Thus, if the two sets of findings are related, it is not simply because a local reduction in grey matter volume leads to a secondary reduction in the volume of the tracts into which that region's neurons project. An alternative possibility is that the larger volume of white matter in these areas reflects a response to an abnormal reduction in grey matter volume. The findings could also be considered as reflective of increased prefrontal cortical folding as measured by the gyrification index, which can be seen as an indirect measure of axonal connectivity. ${ }^{38}$ Increased prefrontal gyrification index has been demonstrated in established schizophrenia ${ }^{39}$ and has been shown to be predictive of transition to psychosis in cohorts defined as high risk owing to genetic factors, ${ }^{38,40}$ although in the high-risk group, gyrification was not predicted by prefrontal white matter volume $e^{40}$ and the relationship between these two measures remains unclear.

\section{Longitudinal changes}

Comparison of MRI data acquired from participants after they had developed psychosis with data from their earlier baseline scans showed that there was a reduction in white matter volume at a point close to the left fronto-occipital fasciculus. In our previous longitudinal comparison of regional grey matter in the same individuals, we had found a reduction in the volume of the left medial temporal, left orbitofrontal, and cingulate cortices, ${ }^{8}$ so we predicted that there would be white matter changes in tracts connecting these areas. The fronto-occipital fasciculus carries fibres connecting the frontal cortex with the temporal, parietal and occipital cortices of the same hemisphere. ${ }^{34,35}$ The region of reduced white matter volume may also have included fibres to and from the adjacent retrosplenial cingulate gyrus. The white matter changes in this region could thus have been related to the reductions in frontal, temporal and cingulate grey matter volume seen in the same individuals. As there were no significant longitudinal reductions in white matter volume in the participants who did not develop psychosis, these changes may be related to the transition to psychosis, as opposed to a non-specific time effect.

In both subgroups of participants there were longitudinal increases in the volume of white matter in the posterior parts of the cerebellum. These were more extensive in the participants who developed psychosis, and were bilateral, whereas in the group who remained non-psychotic, the changes were restricted to the left side. In our previous study of the grey matter in these individuals, both subgroups showed reductions in cerebellar volume at follow-up, and the grey matter changes in both cases were left lateralised and inferior to those in the white matter. Nevertheless, the co-occurrence of longitudinal differences in both white and grey matter volume in the same structure suggests that both the grey and white matter in this part of the cerebellum are progressively changing in young adults with an at-risk mental state, but are not specifically related to the subsequent onset of 
psychosis. We cannot comment on whether similar changes would be evident in healthy volunteers, as we did not include this group in our study. Normally, global white matter volume continues to increase until the sixth decade, whereas grey matter volume decreases. ${ }^{41,42}$ The greater magnitude of the changes in cerebellar white and grey matter volume in the group developing psychosis suggests that although these are not specific to psychosis, its onset may be associated with an acceleration of a process involved in normal brain development. Data from patients with very-earlyonset schizophrenia suggests that the normal age-related reduction seen in frontal and parietal grey matter ${ }^{41}$ may be accelerated in early illness in these regions, ${ }^{43}$ and changes in white matter in tracts connecting these zones may be reflective of, or occur in concert with, these changes.

\section{Limitations}

A number of caveats noted in our original grey matter study ${ }^{8}$ remain, including the effects of medication, diagnostic issues and the methodology of voxel-based approaches. It is possible that the longitudinal effects seen in this study in those individuals who developed psychosis are medication-related. Evidence has slowly mounted suggesting medication effects on brain volume, most particularly a differential effect of typical and atypical antipsychotics on grey matter volume. ${ }^{44}$ Less robust evidence exists for similar changes in white matter volume, ${ }^{45}$ although a recent study suggests that this differential effect is matched in white matter in the frontal lobe with atypical antipsychotics increasing and typical antipsychotics decreasing white matter volume. ${ }^{46}$ Given the longitudinal reductions in white matter volume seen in people who later developed psychosis, and that all of these patients bar one were treated with atypical antipsychotics, medication alone would not seem to be able to explain these findings, although the effect of antipsychotics on brain structure and their interaction with the pathological processes presumed to be occurring in schizophrenia are far from resolved.

Not all of the patients who developed psychosis met criteria for a schizophrenia-spectrum disorder and thus these findings may not be specific to schizophrenia. It has been suggested that affective psychoses are biologically more similar to than they are different from schizophrenia-spectrum psychoses, ${ }^{47}$ although our findings in other studies suggest that there are key differences between them. ${ }^{48}$ The numbers in this study did not permit differentiation of diagnostic groups with regard to these brain changes. Additionally, the non-psychosis group should not be considered a 'normal' or control group, as half of these particpants later developed a mental disorder. ${ }^{8}$

Finally, the methodology of voxel-based approaches in examining between-group differences in brain volume has been subject to some criticism, particularly as regards the possible errors introduced by registration, ${ }^{49,50}$ and smoothing protocols. ${ }^{51}$ By using dual-echo (combined proton density and $\mathrm{T}_{2}$ images) to enhance segmentation, we would argue that this attenuates potential registration errors and since the permutation method we have employed does not require smoothing, ${ }^{27}$ the potential displacement introduced by the smoothing kernel is not an issue in this data-set. In addition, although examination of white matter avoids the possible effect of inter-person variability in gyrification, introducing type I error in regions of high cortical variability in unsmoothed data, significant inter-person variability also exists in white matter structures, including the fronto-occipital fasciculi, ${ }^{52}$ which is not detectable by volumetric imaging (where multiple distinct and/or orthogonal tracts may appear as one homogeneous structure), meaning that precise localisation of the changes described is problematic.
This is the first study of white matter volume in people at ultra-high risk of psychosis. The data suggest that, within the 'at-risk' group, the anatomy of the white matter is different according to the subsequent clinical outcome, particularly in the frontal lobe. There also appear to be progressive focal reductions in white matter volume in association with the transition to psychosis and these may reflect changes in the left fronto-occipital fasciculus that may be occurring in intimate association with changes to the cortical regions they connect.

Mark Walterfang, FRANZCP, Melbourne Neuropsychiatry Centre, Department of Psychiatry, University of Melbourne, Neuropsychiatry Unit, Royal Melbourne Hospital, and North Western Mental Health Program, Sunshine Hospital and Royal Melbourne Hospital, Melbourne; Philip K. McGuire, PhD, MRCPsych, Institute of Psychiatry, King's College London, University of London, UK; Alison R. Yung, MD, FRANZCP, King's College London, University of London, UK; Alison R. Yung, MD, FRANZCP,
ORYGEN Research Centre, Early Psychosis Prevention and Intervention Centre ORYGEN Research Centre, Early Psychosis Prevention and Intervention Centre
(EPPIC), Personal Assistance and Crisis Evaluation (PACE) Clinic, and Department of Psychiatry, University of Melbourne, Melbourne; Lisa J. Phillips, PhD, Department of Psychology, University of Melbourne; Dennis Velakoulis, FRANZCP, Melbourne Neuropsychiatry Centre, Department of Psychiatry, University of Melbourne, Neuropsychiatry Unit, Royal Melbourne Hospital, and North Western Mental Health Program, Sunshine Hospital and Royal Melbourne Hospital, Melbourne; Stephen J. Wood, PhD, Melbourne Neuropsychiatry Centre, Department of Psychiatry, University of Melbourne, and North Western Mental Health Program, Sunshine Hospital and Royal Melbourne Hospital, Melbourne; John Suckling, PhD, Brain Mapping Unit, Department of Psychiatry, University of Cambridge, Cambridge, UK; Edward T. Bullmore, PhD, MRCPsych, Brain Mapping Unit, Department of Psychiatry, University of Cambridge, Cambridge; Warrick Brewer, PhD ORYGEN Research Centre, EPPIC, PACE Clinic, and Department of Psychiatry, Univeristy of Melbourne, Melbourne: Bridget Soulsby, BSc, Melbourne Neuropsychiatry Centre, Department of Psychiatry, University of Melbourne, and North Western Mental Health Program, Sunshine Hospital and Royal Melbourne Hospital, Melbourne; Patricia Desmond MSC, FRACR, Department of Radiology, Royal Melbourne Hospital, Melbourne; Patrick D. McGorry, PhD, FRANZCP, ORYGEN Research Centre, EPPIC, PACE Clinic, and Department of Psychiatry, University of Melbourne, Melbourne; Christos Pantelis, MD, MRCPsych, FRANZCP, Melbourne Neuropsychiatry Centre, Department of Psychiatry, University of Melbourne, and North Western Mental Health Program, Sunshine Hospital and Royal Melbourne Hospital, Melbourne, Australia

Correspondence: Dr Mark Walterfang, Melbourne Neuropsychiatry Centre, Level 2, John Cade Building, Royal Melbourne Hospital, 3050 Australia. Email: mark.walterfang@mh.org.au

First received 26 Jul 2007, final revision 28 Jan 2008, accepted 5 Feb 2008

\section{Acknowledgements}

This research was supported by project grants from the National Health and Medical Research Council (NHMRC; grant ID numbers: 970598, 981112), the Stanley Foundation, the lan Potter Foundation, the Woods Family Trust, and program grants from the NHMRC (number 350241) and the Victorian Health Promotion Foundation. M.W. was supported by a Stanley Research Centre Grant and a Pfizer Neuroscience research grant; D.V. and S.J.W. were supported as Research Officers with funding from the NHMRC; P.D.M. was supported by a NARSAD Distinguished Investigator Award. Software development was supported by a Human Brain Project grant from the National Institute of Mental Health, and the National Institute of Biomedical Imaging \& Bioengineering. M.W. takes responsibility for the integrity of the data and the accuracy of the data analysis. All authors had full access to all the data in the study.

\section{References}

1 Shenton M, Dickey C, Frumin M, McCarley R. A review of MRI findings in schizophrenia. Schizophr Res 2001; 49: 1-52.

2 Weinberger D, Mcclure R. Neurotoxicity, neuroplasticity and magnetic resonance imaging morphometry: what is happening in the schizophrenic brain? Arch Gen Psychiatry 2002; 59: 553-8.

3 Velakoulis D, Pantelis C, McGorry PD, Dudgeon P, Brewer W, Cook M, Desmond P, Bridle N, Tierney P, Murrie V, Singh B, Copolov D. Hippocampal volume in first-episode psychosis and chronic schizophrenia: a highresolution magnetic resonance imaging study. Arch Gen Psychiatry 1999; 56: $133-40$.

4 Roy PD, Zipursky RB, Saint-Cyr JA, Bury A, Langevin R, Seeman MV. Temporal horn enlargement is present in schizophrenia and bipolar disorder. Biol Psychiatry 1998; 44: 418-22.

5 Cahn W, Hulshoff Pol HE, Lems EB, van Haren NE, Schnack HG, van der Linden JA, Schothorst PF, van Engeland $H$, Kahn RS. Brain volume changes in first-episode schizophrenia: a 1-year follow-up study. Arch Gen Psychiatr 2002; 59: 1002-10.

6 Kasai K, Shenton ME, Salisbury DF, Hirayasu Y, Lee CU, Ciszewski AA Yurgelun-Todd D, Kikinis R, Jolesz FA, McCarley RW. Progressive decrease of 
left superior temporal gyrus gray matter volume in patients with first-episode schizophrenia. Am J Psychiatry 2003; 160: 156-64.

7 van Haren NE, Hulshoff Pol HE, Schnack HG, Cahn W, Mandl RC, Collins DL, Evans AC, Kahn RS. Focal gray matter changes in schizophrenia across the course of the illness: a 5-year follow-up study. Neuropsychopharmacol 2007 32: 2057-66.

8 Pantelis C, Velakoulis D, McGorry PD, Wood SJ, Suckling J, Phillips L, Yung AR, Bullmore ET, Brewer W, Soulsby B, Desmond P, McGuire PK. Neuroanatomical abnormalities before and after onset of psychosis: a crosssectional and longitudinal MRI comparison. Lancet 2003; 361: 281-8.

9 Job D, Whalley $H$, McIntosh A, Owens D, Johnstone E, Lawrie S. Grey matter changes can improve the prediction of schizophrenia in subjects at high risk. BMC Med 2006; 4: 29.

10 Job D, Whalley H, Johnstone E, Lawrie S. Grey matter changes over time in high risk subjects developing schizophrenia. Neuroimage 2005; 25: 1023-30.

11 Friston K. Schizophrenia and the disconnection hypothesis. Acta Psychiatr Scand Suppl 1999; 99: 68-79.

12 Friston K, Frith C. Schizophrenia: a disconnection syndrome? Clin Neurosci 1995; 3: 89-97.

13 McGuire $\mathrm{P}$, Frith C. Disordered functional connectivity in schizophrenia. Psychol Med 1996; 26: 663-7.

14 Breir A, Buchanan R, Elkashef A, Munson R, Kirkpatrick B, Gellad F. Brain morphology and schizophrenia: a magnetic resonance imaging study of limbic, prefrontal cortex, and caudate structures. Arch Gen Psychiatry 1992; 49: 921-6.

15 Buchanan R, Vladar K, Barta P, Pearlson G. Structural evaluation of the prefrontal cortex in schizophrenia. Am J Psychiatry 1998; 155: 1049-55.

16 Hulshoff Pol HE, Schnack HG, Bertens MG, van Haren NE, van der Tweel I, Staal WG, Baaré WF, Kahn RS. Volume changes in gray matter in patients with schizophrenia. Am J Psychiatry 2002; 159: 244-50.

17 Paillère-Martinot $M$, Caclin $A$, Artiges $E$, Poline JB, Joliot $M$, Mallet $L$, Recasens C, Attar-Lévy D, Martinot JL. Cerebral gray and white matter reductions and clinical correlates in patients with early onset schizophrenia. Schizophr Res 2001; 50: 19-26.

18 Sanfilipo M, Lafargue T, Rusinek H, Arena L, Loneragan C, Lautin A, Feiner D, Rotrosen J, Wolkin A. Volumetric measure of the frontal and temporal lobe regions in schizophrenia: relationship to negative symptoms. Arch Gen Psychiatry 2000; 57: 471-80.

19 Sigmundsson T, Suckling J, Maier M, Williams S, Bullmore E, Greenwood K, Fukuda R, Ron M, Toone B.. Structural abnormalities in frontal, temporal and limbic regions and interconnecting white matter tracts in schizophrenic patients with prominent negative symptoms. Am J Psychiatry 2001; 158: 234-43.

20 Wible CG, Anderson J, Shenton ME, Kricun A, Hirayasu Y, Tanaka S, Levitt JJ, O'Donnell BF, Kikinis R, Jolesz FA, McCarley RW. Prefrontal cortex, negative symptoms, and schizophrenia: an MRI study. Psychiatry Res 2001; 108: 65-78.

21 Okugawa G, Sedvallo G, Agartz I. Reduced grey and white matter volumes in the temporal lobe of male patients with chronic schizophrenia. Eur Arch Psychiatry Clin Neurosci 2002; 252: 120-3.

22 Takahashi K, Suzuki M, Kawasaki Y, Hagino H, Yamashita I, Nohara S, Nakamura $\mathrm{K}$, Seto $\mathrm{H}$, Kurachi $\mathrm{M}$. Perigenual cingulate gyrus volume in patients with schizophrenia: a magnetic resonance imaging study. Biol Psychiatry 2003; 53: 593-600.

23 Kanaan R, Kim J, Kaufmann W, Pearlson G, Barker G, McGuire P. Diffusion tensor imaging in schizophrenia. Biol Psychiatry 2005; 58: 921-9.

24 Walterfang $\mathrm{M}$, Wood S, Velakoulis D, Pantelis C. Neuropathological, neurogenetic and neuroimaging evidence for white matter pathology in schizophrenia. Neurosci Biobehav Rev 2006; 30: 918-48.

25 Yung AR, Phillips LJ, McGorry PD, McFarlane CA, Francey S, Harrigan S, Patton GC, Jackson HJ. Prediction of psychosis. A step towards indicated prevention of schizophrenia. Br J Psychiatry 1998; 172 (suppl 33): 14-20.

26 Yung AR, Stanford C, Cosgrave E, Killackey E, Phillips L, Nelson B, McGorry $\mathrm{PD}$. Testing the ultra-high risk (prodromal) criteria for the prediction of psychosis in a clinical sample of young people. Schizophr Res 2006; 84 : 57-66.

27 Bullmore ET, Suckling J, Overmeyer S, Rabe-Hesketh S, Taylor E, Brammer MJ. Global, voxel and cluster tests, by theory and permutation for a difference between two groups of structural MR images of the brain. IEEE Trans Med Imaging 1999; 18: 32-42.

28 Suckling J, Sigmundsson T, Greenwood K, Bullmore E. A modified fuzzy clustering algorithm for operated independent tissue classification of dual echo MR images. Magn Reson Imaging 1999; 17: 1065-76.

29 suckling J, Davis MH, Ooi C, Wink AM, Fadili J, Salvador R, Welchew D, Sendur L, Maxim V, Bullmore ET. Permutation testing of orthogonal factorial effects in a language processing experiment using fMRI. Hum Brain Mapp 2006; 27: 425-33.

30 Suckling J, Bullmore E. Permutation tests for factorially designed neuroimaging experiments. Hum Brain Mapp 2004; 22: 193-205.

31 Schmahmann JD, Doyon J, McDonald D, Holmes C, Lavoie K, Hurwitz AS, Kabani N, Toga A, Evans A, Petrides M. Three-dimensional MRI atlas of the human cerebellum in proportional stereotaxic space. Neuroimage 1999; 10 233-60.

32 Gloor P. The Temporal Lobe and Limbic System. Oxford University Press, 1997.

33 Petrides M, Pandya D. Association fiber pathways to the frontal cortex from the superior temporal region in the rhesus monkey. J Comp Neurol 1988; 273: 52-66.

34 Crosby E. Correlative Anatomy of the Nervous System. Macmillan, 1962.

35 Catani M, Howard R, Pajevic S, Jones D. Virtual in vivo interactive dissection of white matter fasciculi in the human brain. Neuroimage 2002; 17: 77-94.

36 McGuire P, Bates J, Goldman-Rakic P. Interhemispheric integration: I. Symmetry and convergence of the corticocortical connections of the left and the right principal sulcus (PS) and the left and the right supplementary motor area (SMA) in the rhesus monkey. Cereb Cortex 1991; 1: 390-407.

37 Yeterian E, Pandya D. Thalamic connections of the cortex of the superior temporal sulcus in the rhesus monkey. J Comp Neurol 1989; 282: 80-97.

38 Harris J, Whalley $H$, Yates S, Miller P, Johnstone EC, Lawrie SM. Abnormal cortical folding in high-risk individuals: a predictor of the development of schizophrenia. Biol Psychiatry 2004; 56: 182-9.

39 Vogeley K, Tepest R, Pfeiffer U, Schneider-Axmann T, Maier W, Honer WG, Falkai $P$. Right frontal hypergyria differentiation in affected and unaffected siblings of families multiply affected with schizophrenia: a morphometric MRI stdy. Am J Psychiatry 2001; 158: 494-6.

40 Harris J, Moorhead T, Miller P, McIntosh A, Bonnici H, Owens D, Johnstone EC, Lawrie SM. Increased prefrontal gyrification in a large high-risk cohort characterizes those who develop schizophrenia and reflects abnormal prefrontal development. Biol Psychiatry 2007; 62: 722-9.

41 Sowell E, Peterson B, Thompson P, Welcome S, Henkenius A, Toga A Mapping cortical change across the human life span. Nat Neurosci 2003; 6: 309-15.

42 Bartzokis G, Beckson M, Lu PH, Nuechterlein KH, Edwards N, Mintz J. Agerelated changes in frontal and temporal lobe volumes in men: a magnetic resonance imaging study. Arch Gen Psychiatry 2001; 58: 461-5.

43 Thompson PM, Vidal C, Giedd JN, Gochman P, Blumenthal J, Nicolson R, Toga AW, Rapoport JL. Mapping adolescent brain change reveals dynamic wave of accelerated gray matter loss in very early-onset schizophrenia. Proc Natl Acad Sci USA 2001; 98: 11650-5.

44 Lieberman JA, Tollefson GD, Charles C, Zipursky R, Sharma T, Kahn RS, Keefe RS, Green Al, Gur RE, McEvoy J, Perkins D, Hamer RM, Gu H, Tohen M; HGDH Study Group. Antipsychotic drug effects on brain morphology in first-episode psychosis. Arch Gen Psychiatr 2005; 62: 361-70.

45. Molina V, Reig S, Sanz J, Palomo T, Benito C, Sánchez J, Sarramea F, Pascau $\mathrm{J}$, Desco M. Increase in gray matter and decrease in white matter volumes in the cortex during treatment with atypical neuroleptics in schizophrenia. Schizophr Res 2005; 80: 61-71.

46 Bartzokis G, Lu PH, Nuechterlein KH, Gitlin M, Doi C, Edwards N, Lieu C, Altshuler LL, Mintz J. Differential effects of typical and atypical antipsychotics on brain myelination in schizophrenia. Schizophr Res 2007; 93: 13-22.

47 Potash J. Carving chaos: genetics and the classification of mood and psychotic syndromes. Harv Rev Psychiatry 2006; 14: 47-63.

48 Velakoulis D, Wood SJ, Wong MT, McGorry PD, Yung A, Phillips L, Smith D, Brewer W, Proffitt T, Desmond P, Pantelis C. Hippocampal and amygdala volumes differ according to psychosis stage and diagnosis: an MRI study of chronic schizophrenia, first-episode psychosis and ultra-high risk subjects. Arch Gen Psychiatry 2006; 63: 139-49.

49 Bookstein F. Voxel-based morphometry should not be used with imperfectly registered images. Neuroimage 2001; 14: 1454-62.

50 Crum W, Griffin L, Hill D, Hawkes D. Zen and the art of medical image registration: correspondence, homology, and quality. Neuroimage 2003; 20 1425-37.

51 Jones D, Symms M, Cercignani M, Howard R. The effect of filter size on VBM analyses of DT-MRI data. Neuroimage 2005; 26: 546-54.

52 Burgel U, Amunts K, Hoemke L, Mohlberg H, Gilsbach J, Zilles K. White matter fiber tracts of the human brain: three-dimensional mapping at microscopic resolution, topography and intersubject variability. Neuroimage 2005; 29 : 1092-105.

53 Cogan D, Chu F, Reingold D, Barranger J. Ocular motor signs in some metabolic diseases. Arch Opthalmol 1981; 99: 1802-8. 